\title{
Depression is a treatable cause of suffering among multiple sclerosis patients and can result in suicide
}

\author{
Dennis Bourdette, $M D$
}

Depression results from chemical changes within the brain and results in feeling sad, excessive tiredness, loss of interest in daily activities, and changes in eating and sleeping habits. Severe depression can lead to thoughts of suicide and is the most common problem leading to suicide. Importantly, depression is usually treatable with medications and psychotherapy.

Depression often complicates multiple sclerosis (MS). Estimates vary, but 50 to $60 \%$ of MS patients develop some degree of depression and, like depression in general, it responds to treatment. While MS is often disabling, it rarely causes death. One cause of premature death among MS patients is suicide, which occurs more frequently in these patients than in the general population. You can learn more about depression and MS on the next page.

In this issue of Neurology, two articles (Feinstein A. An examination of suicidal intent in patients with multiple sclerosis. Neurology 2002;59: 674-678; and Patten SH, Metz LM. Interferon ß-1a and depression in secondary progressive MS: Data from the SPECTRIMS Trial. Neurology 2002;59:744-746) shed new light on the problems of depression and suicide in MS. An editorial (Caine ED, Schwid SR. Multiple sclerosis, depression, and the risk of suicide. Neurology 2002;59:662-663) also appears in this month's Neurology.

Feinstein studied 140 patients attending an MS clinic in Toronto, Canada. He found that 40 (29\%) patients had thought about committing suicide and nine had at-
Table Things that do and do not affect the risk of suicidal thoughts among $M S$ patients

Things that increase the risk of suicidal thoughts among MS patients

- Major depression

- Living alone

- Alcohol abuse

Things that do not affect the risk of suicide among MS patients

- Duration of MS

- Severity of physical disability

- Cognitive impairment

tempted suicide. Among the entire group of 140 patients, about onethird had a history of major depression at some time. In comparing the 40 patients who had a history of suicidal intent with the 100 who did not, Feinstein discovered that there were no differences between the two groups in age, sex, duration or type of MS, physical disability, or cognitive impairment. However, the group with a history of suicidal intent were much more likely to have problems with depression. About one-third of the suicidal group had evidence of significant depression at the time of study, compared with only $2 \%$ in the nonsuicidal group. In addition, $83 \%$ of the suicidal group had a history of major depression at some time in their lives compared with only $18 \%$ of the nonsuicidal group.

There were several factors that seemed to predict suicidal intent, including having a history of living alone, a family history of mental illness, significant depression or anxiety at the time of testing, significant psychosocial stress, and a diagnosis at any time of major depression, anxiety disorder, or alcohol abuse. The three most important factors were living alone, severe depression, and history of alcohol abuse.

The study by Patten and Metz addressed the concern that a commonly used treatment for MS, $\beta$-interferon, might cause depression as a side effect. As part of a 3-year study comparing placebo vs one type of $\beta$-interferon, Patten and Metz assessed 365 MS patients every 6 months for depression. They found that patients receiving $\beta$-interferon were no more likely to be depressed than those receiving placebo, indicating that depression is not a common side effect of $\beta$-interferon treatment. They discovered that depression among MS patients fluctuates over time. This suggests that it is important for physicians caring for MS patients to evaluate for depression at each clinic visit.

This is important because severe depression among MS patients often goes untreated. About one-third of MS patients that experience major depression or have suicidal thoughts never receive treatment for depression. MS patients with depression respond to treatment with antidepressants and psychotherapy. Depression is therefore a treatable cause of suffering among MS patients. Because it increases the risk of suicide, appropriate treatment can decrease the risk of MS patients committing suicide. It is very important for physicians to recognize and treat depression among their MS patients. Patients and family members also should report depressive symptoms to their physicians to aid in appropriate treatment. 


\section{About Multiple Sclerosis}

MS is a disabling neurologic disorder of young adults, affecting at least 300,000 Americans. The average age at diagnosis is 30, typically starting between the ages of 15 and 50. Women are affected at least twice as often as men. It is more common in persons of northern European heritage and those living furthest from the equator.

\section{MS involves inflammation within} the central nervous system (the brain and spinal cord), followed by the loss of the protective myelin sheaths that surround nerve fibers. When the myelin is damaged, nerve impulses are not quickly and efficiently transmitted. Besides damage to the myelin sheaths, it is now recognized that the nerve fibers, called axons, also are damaged in MS to varying extent. Lesions (called plaques) develop in the brain and spinal cord and can cause the symptoms of MS listed below.

\section{What Are the Symptoms?}

There are several types of MS. Most people with MS begin with relapsing-remitting disease. This means that the symptoms come and go, often leaving the person feeling nearly normal until another relapse, or MS attack, occurs.

Symptoms associated with relapses usually develop over a period of days. The problems can last for a matter of days or weeks and then go away, sometimes even without any treatment. New attacks occur at irregular intervals, usually one attack every 1 to 2 years.

Common symptoms include:

- Vision loss

- Numbness or tingling

- Weakness or fatigue

- Unsteadiness in walking

- Double vision
- Heat intolerance

- Partial or complete paralysis

- Electric shock sensations when bending the neck

About $50 \%$ of patients with relapsing-remitting MS develop a progressive form of MS, called secondary progressive MS, in which there is continual worsening. In this phase of the disease, patients may continue to have relapses or may stop having them altogether. About $15 \%$ of patients have progressive worsening from the beginning of their MS and do not experience relapses of MS. This form of MS is called primary progressive MS.

\section{How Is MS Diagnosed?}

The diagnosis of MS is based on a history of multiple attacks over time of neurologic lesions that affect different parts of the central nervous system. Your neurologist will order tests that will help confirm the diagnosis. Usually a magnetic resonance imaging (MRI) scan of the brain (and possibly the spinal cord) is ordered to find evidence of abnormal areas. Lumbar puncture (spinal tap) is also helpful to detect specific problems with the cerebrospinal fluid.

\section{What Causes Multiple Sclerosis?}

The cause of MS is unknown.

There is strong evidence that MS is immune mediated. This means that the person's own immune system attacks the central nervous system (an autoimmune disease).

\section{What Are the Treatments?}

Currently, there is no prevention or cure for MS. However, this is a promising time for people with MS, as several new medications that affect the underlying disease process have been approved or are awaiting approval by the US Food and Drug Administration (FDA). You should ask your neurologist about the best treatment options for you. Current treatments are divided into three categories:

Medications that treat the symptoms of MS. These include medications to treat depression, decrease muscle stiffness, reduce fatigue, control bladder symptoms, reduce pain, and address sexual dysfunction.

Medications that modify attacks when they occur. These are primarily corticosteroids (a synthesized adrenal hormone) that can shorten an attack.

Medications that modify disease activity. These are taken on a regular basis to help reduce the frequency of attacks and the longterm damage to brain caused by MS. Disease-modifying therapies currently FDA-approved for treating MS include recombinant $\beta$-interferons (Avonex, Betaseron, and Rebif), glatiramer acetate (Copaxone), and an immunosuppressant/chemotherapy drug, mitoxantrone (Novantrone).

\section{For More Information:}

National Multiple Sclerosis Society

733 Third Avenue

New York, NY 10017

800-FIGHT-MS

Multiple Sclerosis Association of America

601 White Horse Pike

Oaklyn, NJ 08107

800-333-4MSA

American Academy of Neurology
1080 Montreal Avenue
St. Paul, MN 55116
Tel: $800-879-1960$
Fax: $651-695-2791$
http://www.aan.com

American Academy of Neurology Education \& Research Foundation

1080 Montreal Avenue

St. Paul, MN 55116

651-695-2712

http://www.foundation@aan.com

http://www.thebrainmatters.org 


\section{Neurology}

Depression is a treatable cause of suffering among multiple sclerosis patients and can result in suicide

Dennis Bourdette

Neurology 2002;59;E6-E7

DOI 10.1212/WNL.59.5.E6

This information is current as of September 10, 2002

Updated Information \&

Services

Permissions \& Licensing

Reprints including high resolution figures, can be found at: http://n.neurology.org/content/59/5/E6.full

Information about reproducing this article in parts (figures,tables) or in its entirety can be found online at:

http://www.neurology.org/about/about_the_journal\#permissions

Information about ordering reprints can be found online:

http://n.neurology.org/subscribers/advertise

Neurology $®$ is the official journal of the American Academy of Neurology. Published continuously since 1951, it is now a weekly with 48 issues per year. Copyright . All rights reserved. Print ISSN: 0028-3878. Online ISSN: 1526-632X.

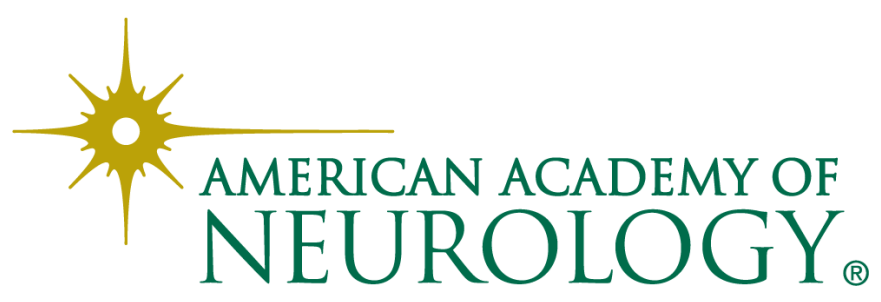

